Cómo citar este artículo: Castañeda, S y Caiaffa, N. (2015, enero-julio). Relación entre la composición corporal y el rendimiento físico en la Escuela Militar de Cadetes José María Córdova. Rev. Cient. Gen. José María Córdova 13(15), 257-270

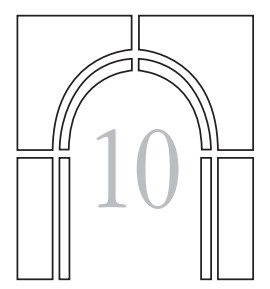

\title{
Relación entre la composición corporal y el rendimiento físico en la Escuela Militar de Cadetes José María Córdova*
}

Recibido: 11 de noviembre de 2014 • Aceptado: 10 de enero de 2015

\section{Relationship Between Body Composition and Physical Performance of the Colombian Military Academy}

Relation entre la composition corporelle et la performance physique à l’École militaire des cadets "Général José María Córdova »

\section{Relação entre composição corporal e desempenho físico na Escola Militar de Cadetes General José María Córdova}

Sergio Mauricio Castañeda Tovar a

Ninosca Sofía Caiaffa Bermúdez ${ }^{b}$

* Articulo asociado al proyecto de investigación: Análisis del rendimiento físico y la composición corporal en Cadetes y Alféreces de la Escuela Militar de Cadetes José María Córdova (Código CICFI-01.2014); aprobado por la dirección de investigaciones de la Escuela Militar de Cadetes "General José María Córdova” y financiado por la misma institución en el grupo de investigación RENFIMIL (Rendimiento físico militar). Bogotá D.C., Colombia.

a Licenciado en Educación Física de la Universidad Pedagógica Nacional; Magíster en Pedagogía de la Cultura Física de la Universidad Pedagógica y Tecnológica de Colombia-UPTC. Autor para correspondencia. Contacto: profesergio15@gmail.com

b Nutricionista y dietista del deporte de la Universidad del Atlántico; Antropometrista certificada por The International Society for the Advancement of Kinanthropometry Level II. Contacto: ncaiaffabermudez@gmail.com 
Resumen. El rendimiento físico y la composición corporal son elementos clave en la desarrollo de la labor operacional del Militar del Ejército de Colombia. El objetivo de este estudio fue establecer la relación entre la composición corporal y el rendimiento físico en los estudiantes de la Escuela Militar de Cadetes General José María Córdova. Se realizó un estudio descriptivo-correlacional donde se valoraron 51 estudiantes del género masculino (cabe aclarar que todos firmaron los consentimientos informados), quienes realizan el proceso de formación en Ciencias Militares en la ciudad de Bogotá D.C. Las características de la población muestran que la edad promedio es de 19,45 años, la estatura se ubicó en 172,6 cm, la masa corporal obtuvo $68,33 \mathrm{~kg}$ y el índice de masa corporal se ubica en 22,97. Se analizaron las variables: en primer lugar rendimiento físico, la cual fue evaluada por medio de la directiva 300-7 de 2013 de la JEDOC, valorando el test de navette $(9,7)$, el test de Wells, el test de barras $(8,4)$, los test en un minuto de abdominales $(76,7)$ y flexiones de codo $(54,8)$. La segunda variable, composición corporal, fue determinada a través del modelo tetra comportamental, propuesto por De Ross Guimaraes, resultando la caracterización de la tipología de la población con un 2,0\% meso-endomorfo, 49,0\% endo-mesomorfo, 15,7\% ecto-mesomorfo, 5,9\% meso-ectomorfo y $27,5 \%$ mesomorfo balanceado.

Palabras clave: antropometría, composición corporal, cualidades físicas, militares, test físicos.

Abstrac. Physical performance and body composition are key elements in the development of the operational work of the Army Military Colombia. The aim of this study was to establish the relationship between body composition and physical performance in students of the Military School of Cadets General José María Córdova a -correlacional sectional study with 51 male students were assessed was performed, all signed the informed consent, which perform the training process in Military Sciences in Bogotá. The characteristics of the population show that the average age is 19.45 years, height stood at $172.6 \mathrm{~cm}$, body mass gained $68.33 \mathrm{~kg}$, the BMI is at 22.97. Physical performance variables which was assessed by the Directive 300-7 of 2013 JEDOC were analyzed, evaluating test navette (9.7), the test Wells, the test bars $(8,4)$ and the test in one minute abdominal $(76.7)$ and elbow bends $(54.8)$. Body composition was determined by varying proposed by De Guimaraes resulting Ross characterizing the type of population with $2.0 \%$ meso-endomorfo, endo-mesomorfo $49.0 \%, 15.7 \%$ ecto-mesomorfo, $5.9 \%$ meso-ectomorfo tetra-comportamental model $27.5 \%$ balanced mesomorph.

Key words: anthropometry, body composition, military, physical attributes, physical test.

Resumo: $\mathrm{O}$ condicionamento físico e a composição corporal são elementos fundamentais para o desenvolvimento da atividade operacional do militar do Exército Colombiano. O objetivo deste estudo foi estabelecer a relação entre a composição corporal e o condicionamento físico dos alunos da Escola Militar de Cadetes General José María Córdova. Foi realizado um estudo descritivo-correlativo com 51 alunos do gênero masculino que atualmente encontram-se no processo de formação em Ciências Militares, na cidade de Bogotá, em que todos concordaram com a realização deste estudo, por meio do termo de consentimento. As características da amostra são as seguintes: idade média de 19,45 anos, estatura média de 172,6 cm, massa corporal de 68,33 Kg e índice de massa corporal média de 22,97. As variáveis do condicionamento físico foram analisadas por meio da Directiva 300-7 de 2013 da JEDOC, com os seguintes: teste de navette $(9,7)$, o teste de Wells $(\ldots)$, teste de barra $(8,4)$, teste de abdominais em $1 \mathrm{~min}(76,7)$, flexão de braços $(54,8)$. A variável composição corporal foi determinada por meio do modelo tetra comportamental de De Ross Guimaraes tendo como resultado da amostra o seguinte: 2,0\% meso-endomorfo, 49,0\% endo mesomorfo, $15,7 \%$ ecto mesomorfo, 5,9\% mesoectomorfo, $27,5 \%$ mesomorfo.

Palavras Chaves: Composicao Corporal, Antropometría, qualidades físicas, teste físico, militares. 


\section{Introducción}

El militar colombiano, dada su labor, debe poseer un rendimiento físico elevado y una composición corporal equilibrada; es por esto que es muy importante lograr niveles de especialización e investigación que busquen identificar y caracterizar estándares e indicadores para esta población en particular.

Las capacidades condicionales tales como la velocidad, la fuerza y la resistencia se encuentran ligadas al desarrollo de la condición física, las cuales se basan en la eficiencia metabólica de los músculos y de la participación de diferentes sistemas (cardio-vascular, respiratorio, sistema nervioso) (Marín, 2012).

Niveles adecuados de aptitud física y de actividad física se vienen señalando como factores de protección para innumerables enfermedades y como promotores de calidad de vida. (Teixeira, et al., 2010). Un alto nivel de condición física está relacionado directamente con un grado óptimo de respuesta fisiológica. La configuración morfológica de un individuo puede ser estudiada mediante su composición corporal y su somatotipo o biotipo, los cuales permiten describir y comparar distintos niveles de rendimiento.

La medición de la composición corporal proporciona una valoración real del nivel físico. La relación de la composición corporal con la salud es aplicada en diversos estudios epidemiológicos donde se definen los beneficios referidos a la escasa adiposidad, aceptable distribución de grasa y la relación entre el exceso con los riesgos de padecer enfermedad aguda o crónica (diabetes, hipertensión arterial, problemas coronarios) (Fajardo 1994). Por ello, determinar la morfología corporal a partir de las variables antropométricas se convierte en un proceso necesario en la valoración del militar, permitiendo realizar comparaciones entre diferentes grupos de grados militares, especialidades y formación en armas; de tal forma que permita optimizar el rendimiento individual y/o colectivo, a fin de plantear programas de entrenamiento que permitan maximizar las posibilidades de conseguir éxitos en los campos estratégicos, tácticos y de combate en la Fuerza.

Un estudio dirigido por Teixeira, et al. (2010) observó la aptitud física, la edad y el estado nutricional en Brasil; Bustamante (2011) analizó la antropometría y composición corporal en Cadetes Navales Argentino; y Fajardo (1994) realizó una valoración nutricional de la ración de campaña en el soldado colombiano. Sin embargo, encontramos escasos referentes teóricos frente a la relación del rendimiento físico y la composición corporal en el militar colombiano.

\section{Metodología}

Este estudio se desarrolló con un enfoque cuantitativo, el tipo de investigación fue descriptiva-correlacional con corte transversal, se emplearon fuentes de información primaria y secundaria, la población estuvo conformada por 51 cadetes y alféreces del género masculino, los cuales se seleccionaron de manera aleatoria en cada una de las compañías orgánicas de la institución.

Como criterios de inclusión era necesario que los cadetes y alféreces permanecieran activos durante el proceso de toma de los datos y cumplieran en su totalidad todas las pruebas de la valoración física. Como criterios de exclusión se tuvo en cuenta que los cadetes y alféreces no presen- 
taran ninguna lesión osteo-muscular durante el proceso y cumplieran el cronograma de toma de datos en antropometría y condición física.

El instrumento de evaluación de la condición física fue la directiva 300-7 de 2013 de la Jefatura de Educación y Doctrina del Ejercito Nacional, la cual incluye dentro de su batería de evaluación el test de Navette, flexión de codos, flexiones abdominales en un minuto, barras y el test de Wells como pruebas básicas.

Para el análisis de la composición corporal se utilizaron los cálculos propuestos por De Ross Guimaraes (Esparza, 1993), siguiendo su modelo tetra compartimental (masa grasa, MG; masa muscular, MM; masa ósea, MO; masa residual, MR). En esta fase se tomaron las medidas antropométricas: peso y talla; pliegues cutáneos del tríceps, subescapular, ileocrestal, supra espinal, abdominal, del muslo y de la pierna; diámetros biestiloideo, biepicondíleo (húmero) y bicondíleo (fémur), así como los perímetros del brazo contraído, muslo y pierna. Todas estas medidas se tomaron por un antropometrísta acreditado nivel II, siguiendo las indicaciones descritas por la International Society for the Avancement in Kineanthropometric (ISAK) (Marfell-Jones, et al., 2006). Se contó con la colaboración de la facultad de educación física militar en el préstamo del antropómetro calibrador óseo lafayete, para la valoración de los diámetros pequeños.

El análisis estadístico se realizó por medio del programa SPSS versión 2.0 destacando las medidas de tendencia central y de dispersión. La normalidad de los datos se confirmó por medio de la prueba de Kolmogorov-Smirnov. Con el propósito de evidenciar posibles asociaciones entre las variables se aplicaron pruebas de correlación de Pearson. Para variables continuas se tuvieron en cuenta, para efecto de análisis de las correlaciones, los criterios adoptados por Malina (1996), los que describieron correlación positiva débil para un valor menor que 0,30 , correlación moderada para valores, entre 0,30 y 0,60 y correlación alta para valores superiores a 0,60.

\section{Resultados}

Tabla 1. Perfil antropométrico de la población

\begin{tabular}{ccccc}
\hline \multicolumn{5}{c}{ Medidas antropométricas básica } \\
\hline \multicolumn{1}{c}{ Ítem } & Media & Desviación típica & Varianza & Asimetría \\
\hline Masa corporal $(\mathrm{kg})$ & 68,33 & 8,75 & 70,69 &, 35 \\
\hline Estatura $(\mathrm{cm})$ & 172,6 & 5,68 & 32,33 &, 92 \\
\hline \multicolumn{5}{c}{ Pliegues cutáneos $(\mathbf{m m})$} \\
\hline Tríceps & 9,69 & 3,08 & 9,52 &, 36 \\
\hline Subescapular & 10,9 & 3,14 & 9,89 &, 96 \\
\hline Cresta iliaca & 16,6 & 6,40 & 41,06 &, 51 \\
\hline Supra espinal & 9,3 & 4,38 & 19,19 &, 33 \\
\hline Abdominal & 16,7 & 7,88 & 19,19 &, 33 \\
\hline Muslo frontal & 12,1 & 4,65 & 21,70 &, 88 \\
\hline Pantorrilla medial & 8,9 & 4,84 & 23,45 & 1,57 \\
\hline
\end{tabular}




\begin{tabular}{|c|c|c|c|c|}
\hline \multicolumn{5}{|c|}{ Medidas antropométricas básica } \\
\hline Ítem & Media & Desviación típica & Varianza & Asimetría \\
\hline \multicolumn{5}{|c|}{ Perímetros (cm) } \\
\hline Brazo en tensión & 32,6 & 2,24 & 5,05 &,- 02 \\
\hline Cintura & 78,6 & 4,22 & 17,88 &, 56 \\
\hline Pantorrilla & 37,0 & 2,60 & 6,80 &, 15 \\
\hline \multicolumn{5}{|c|}{ Diámetros ( cm) } \\
\hline Humero & 6,9 & ,37 &, 14 &,- 24 \\
\hline Femoral & 9,7 &, 52 &, 12 & ,33 \\
\hline Muñeca & 5,7 & ,32 &, 10 &, 54 \\
\hline \multicolumn{5}{|c|}{ Índices } \\
\hline IMC & 22,9 & 2,25 & 5,08 & ,33 \\
\hline Desnutrición & 0 & & & \\
\hline Normal & $82,4 \%(n=42)$ & & & \\
\hline Sobrepeso & $17,6 \%(n=9)$ & & & \\
\hline Obesidad tipo 1 & 0 & & & \\
\hline Obesidad tipo 2 & 0 & & & \\
\hline Obesidad mórbida & 0 & & & \\
\hline Perímetro de cintura & $78,5 \%$ & 4,24 & 18,05 &, 557 \\
\hline Bajo riesgo & $98 \%(n=50)$ & & & \\
\hline Riesgo relativo & $2 \% \quad(n=1)$ & & & \\
\hline Riesgo aumentado. & 0 & & & \\
\hline \multicolumn{5}{|c|}{ Masas corporales } \\
\hline$\%$ de grasa de Yuhasz & 11,0 & 9,90 & 98,02 & 6,58 \\
\hline Masa grasa & 8,0 & 9,14 & 83,70 & 6,46 \\
\hline$\%$ Muscular & 48,8 & 2,00 & 4,02 &,- 01 \\
\hline Masa Muscular & 33,5 & 4,28 & 18,32 &, 43 \\
\hline \% Óseo & 17,5 & 2,83 & 8,05 & $-3,15$ \\
\hline Peso óseo & 11,8 & 1,33 & 1,78 & 1,17 \\
\hline \% Residual & 23,9 &, 58 &, 34 & $-4,89$ \\
\hline Peso residual & 10,5 & $-2,11$ & 4,46 &, 55 \\
\hline
\end{tabular}

Fuente: elaboración propia.

\section{Somatocarta}

La Figura 1 muestra la representación gráfica del somatotipo según la Compañía Orgánica de los cadetes y alféreces. En el análisis comparativo logró determinar que el Somatotipo predominante es el mesomorfo balanceado. 


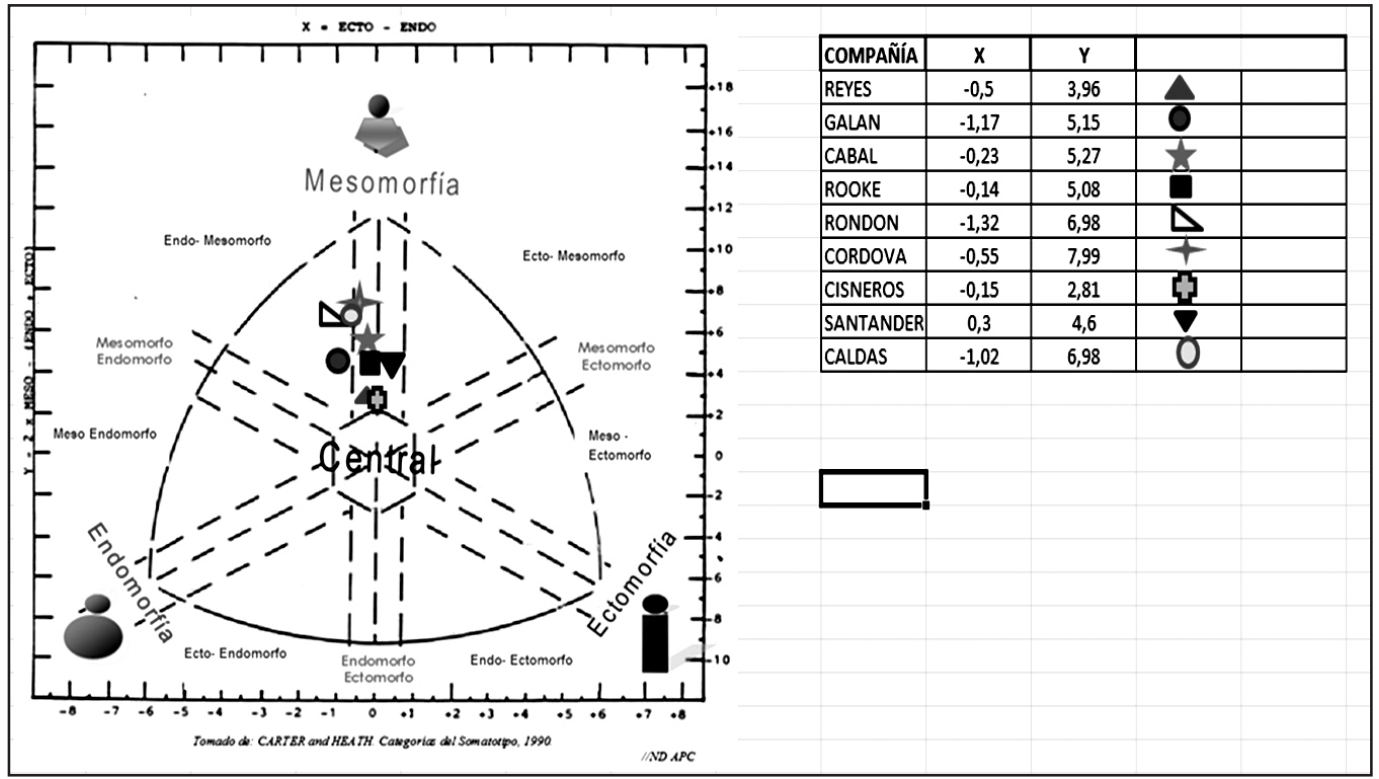

Figura 1. Clasificación por medio de la somatocarta Fuente: elaboración propia.

\section{Capacidades físicas}

Tabla 2. Capacidades físicas

\begin{tabular}{|c|c|c|c|c|c|c|}
\hline \multicolumn{2}{|c|}{ Medida estadística } & Flexión de codo & Abdominal & Navette & Wells & Barras \\
\hline \multicolumn{2}{|c|}{ Media } & 54,88 & 76,76 & 9,72 & 38,84 & 8,43 \\
\hline \multicolumn{2}{|c|}{ Mediana } & 57,00 & 78,00 & 10,00 & 38,00 & 9,00 \\
\hline \multicolumn{2}{|c|}{ Moda } & 60,00 & 78,00 & 11,00 & 38,00 & 10 \\
\hline \multicolumn{2}{|c|}{ Desv. típ. } & 4,89 & 7,25 & 1,34 & 5,44 & 3,21 \\
\hline \multicolumn{2}{|c|}{ Varianza } & 23,98 & 52,66 & 1,80 & 29,61 & 10,33 \\
\hline \multicolumn{2}{|c|}{ Asimetría } &,- 86 &,- 56 &,- 81 & ,08 &,- 79 \\
\hline \multicolumn{2}{|c|}{ Error típ. de asimetría } & ,33 & ,33 & ,33 &, 33 & ,33 \\
\hline \multicolumn{2}{|c|}{ Mínimo } & 40,00 & 58,0 & 6,0 & 28,00 & 0 \\
\hline \multicolumn{2}{|c|}{ Máximo } & 61,00 & 90,0 & 11,00 & 50,00 & 14 \\
\hline \multirow[t]{3}{*}{ Percentiles } & 25 & 50,00 & 71,00 & 9,00 & 35,00 & 7,00 \\
\hline & 50 & 57,00 & 78,00 & 10,00 & 38,00 & 9,00 \\
\hline & 75 & 59,00 & 83,00 & 11,00 & 43,00 & 10,00 \\
\hline
\end{tabular}

Fuente: elaboración propia. 


\section{Nivel de rendimiento físico en los test aplicados}

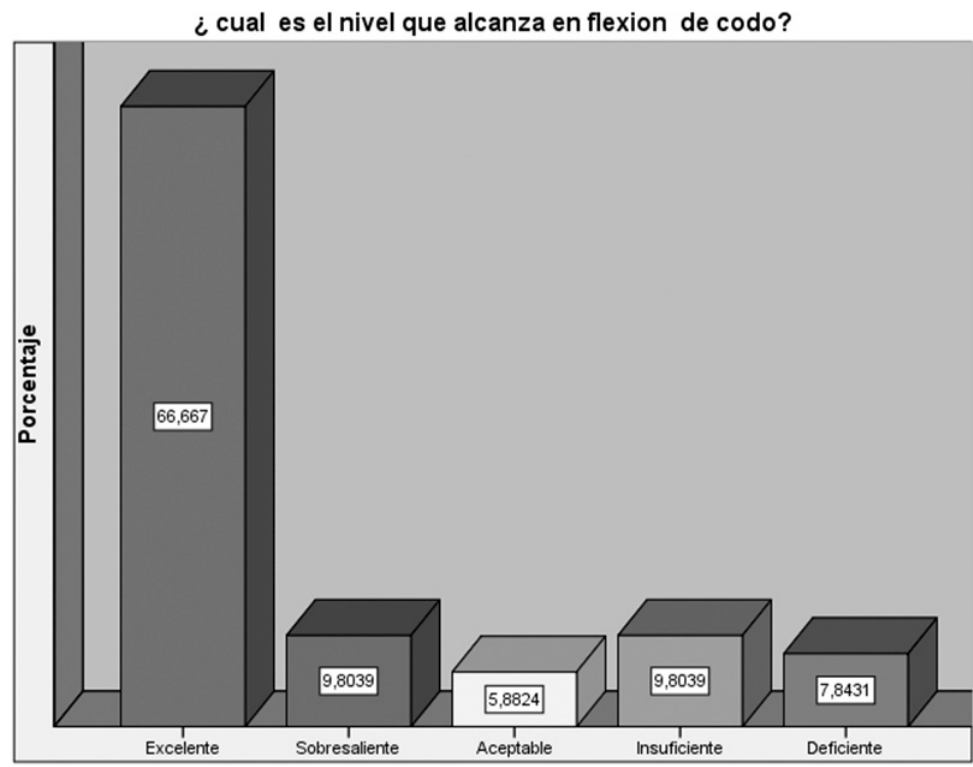

Figura 2. Rendimiento físico en el test flexiones de codo Fuente: elaboración propia.

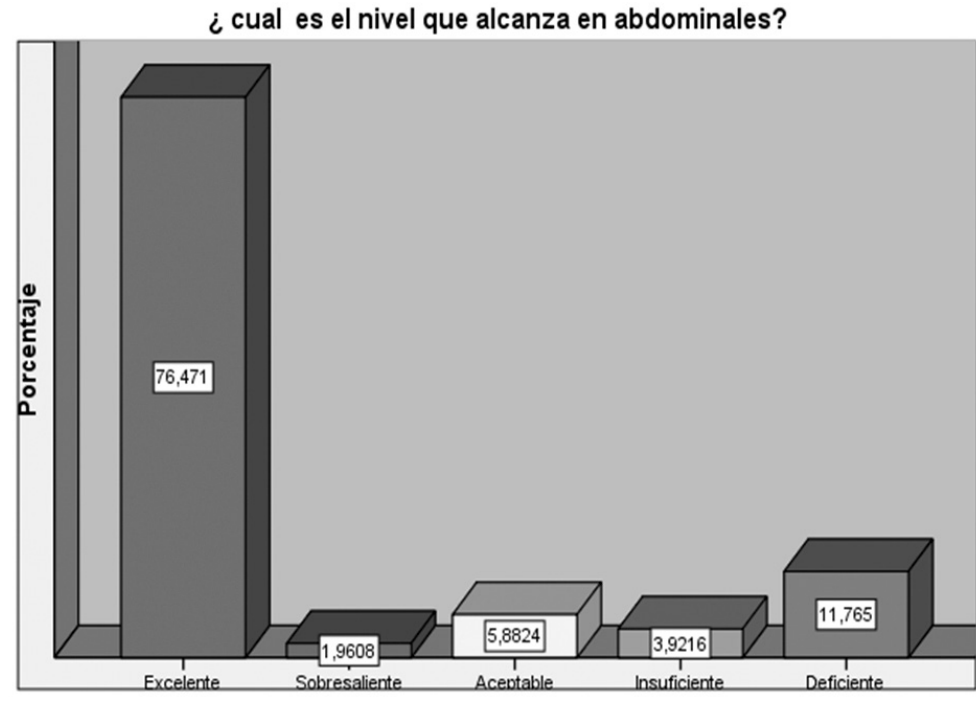

Figura 3. Rendimiento físico en el test de abdominales Fuente: elaboración propia. 


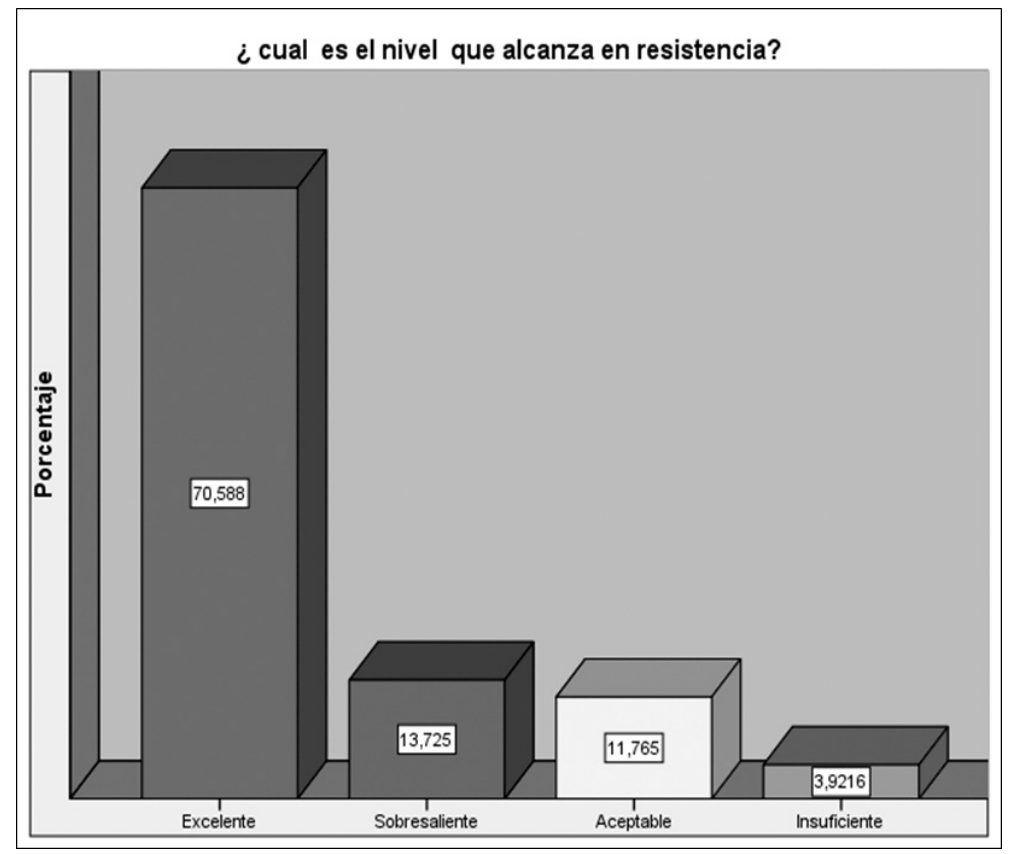

Figura 4. Rendimiento físico en el test Navette Fuente: elaboración propia.

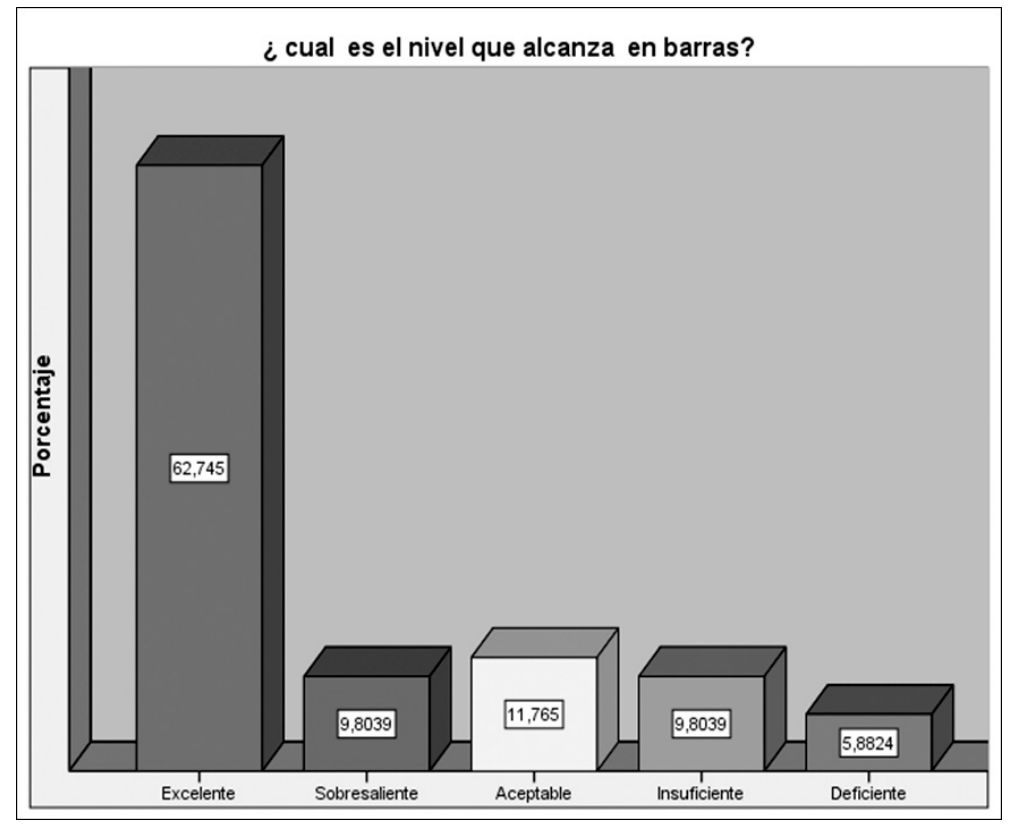

Figura 5. Rendimiento físico en el test de barras Fuente: elaboración propia. 


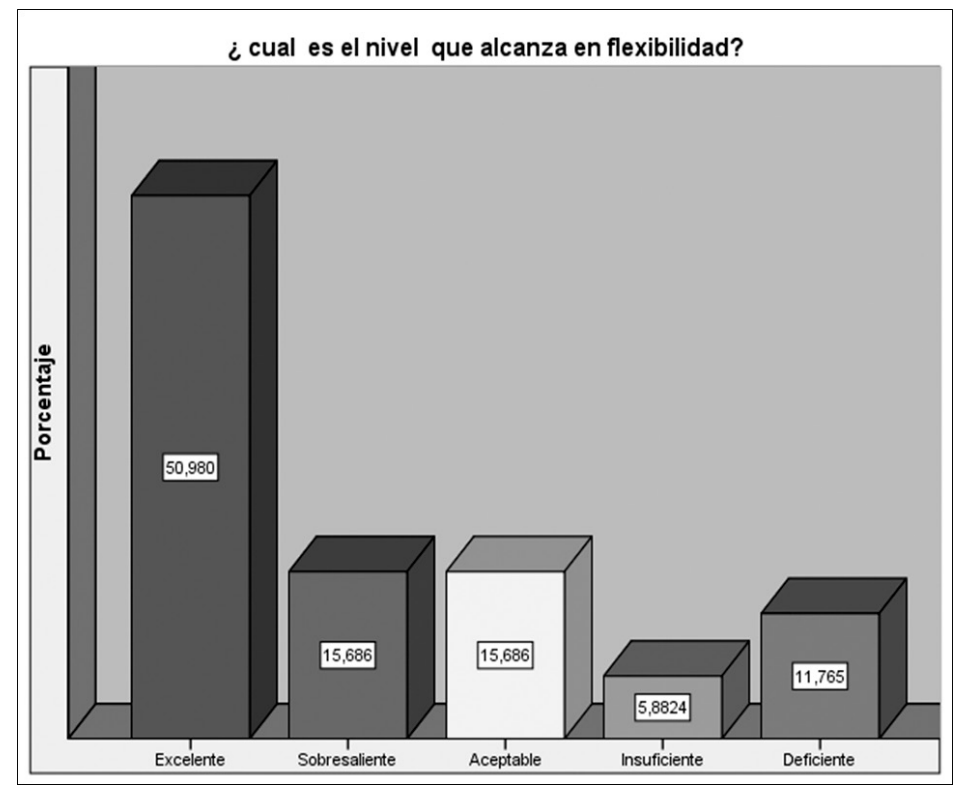

Figura 6. Rendimiento físico en el test de flexibilidad Fuente: elaboración propia.

\section{Niveles de rendimiento y somatotipo (Coeficiente de correlación de Pearson)}

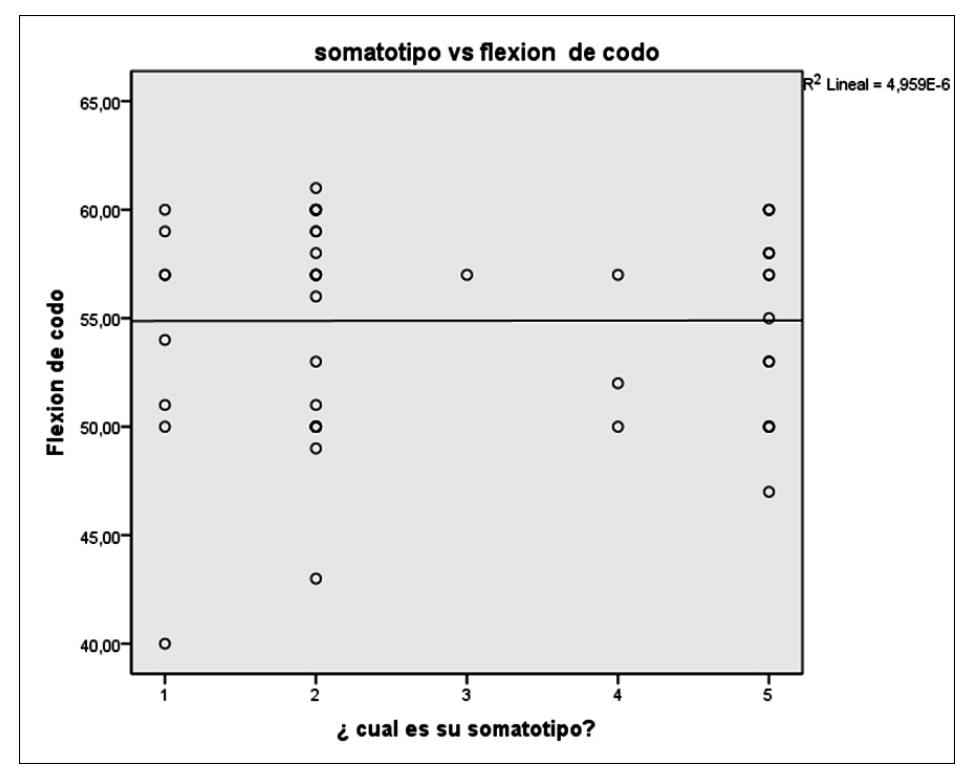

Figura 7. Somatotipo y test de flexión de codo Fuente: elaboración propia. 


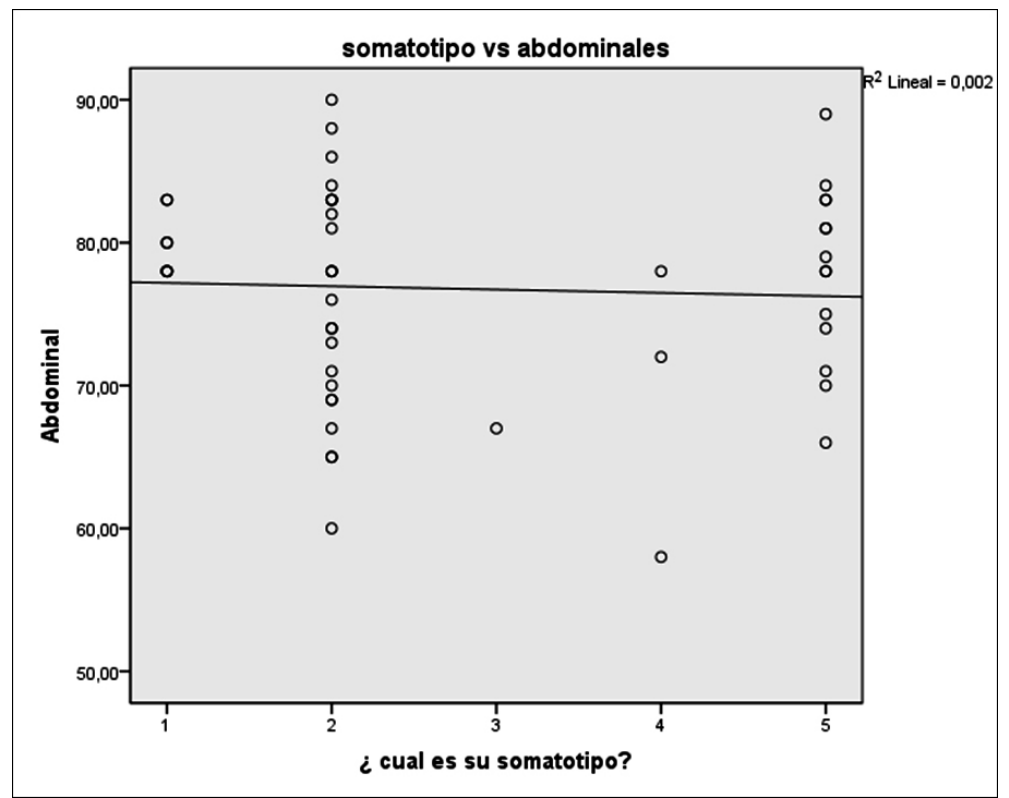

Figura 8. Somatotipo y test de abdominales Fuente: elaboración propia.

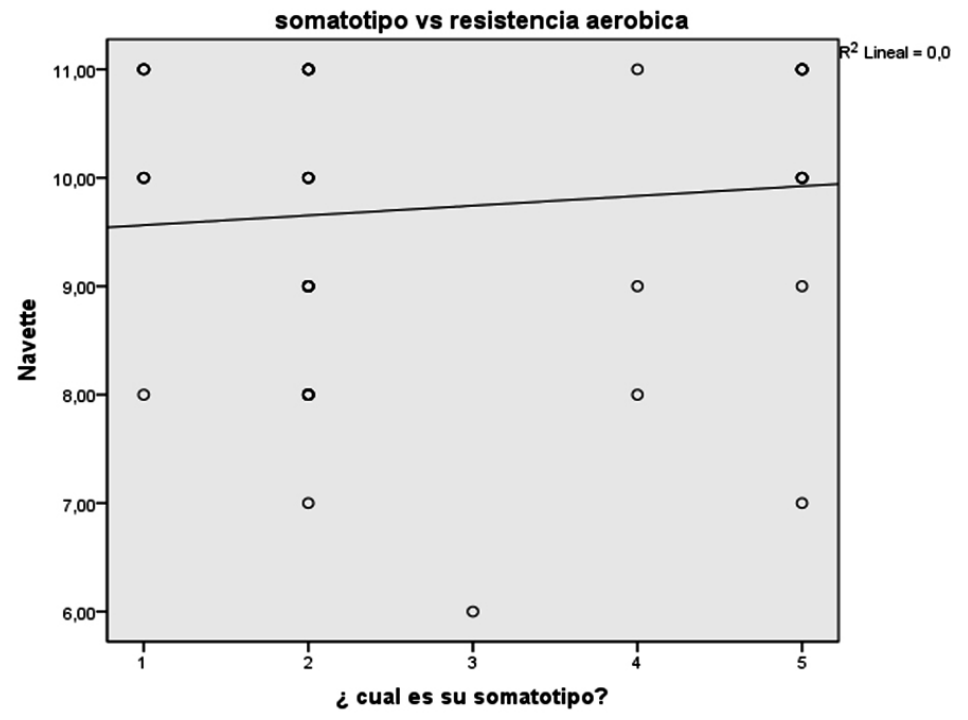

Figura 9. Somatotipo y Vo2 Max

Fuente: elaboración propia. 


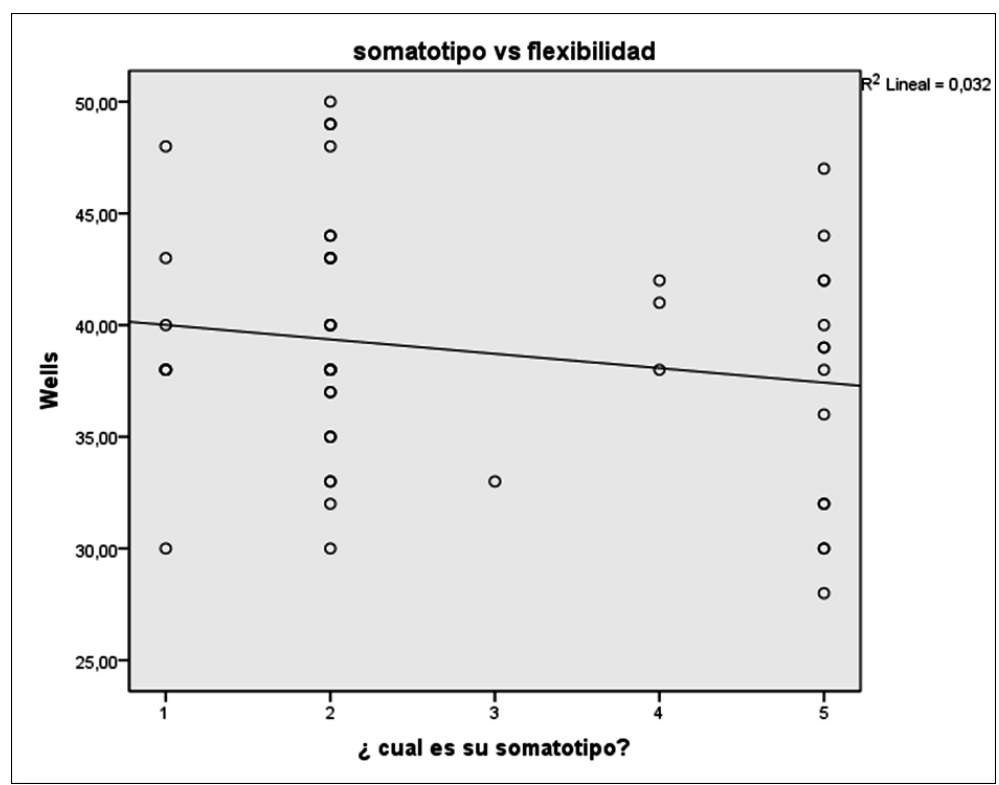

Figura 10. Somatotipo y test de flexibilidad Fuente: elaboración propia.

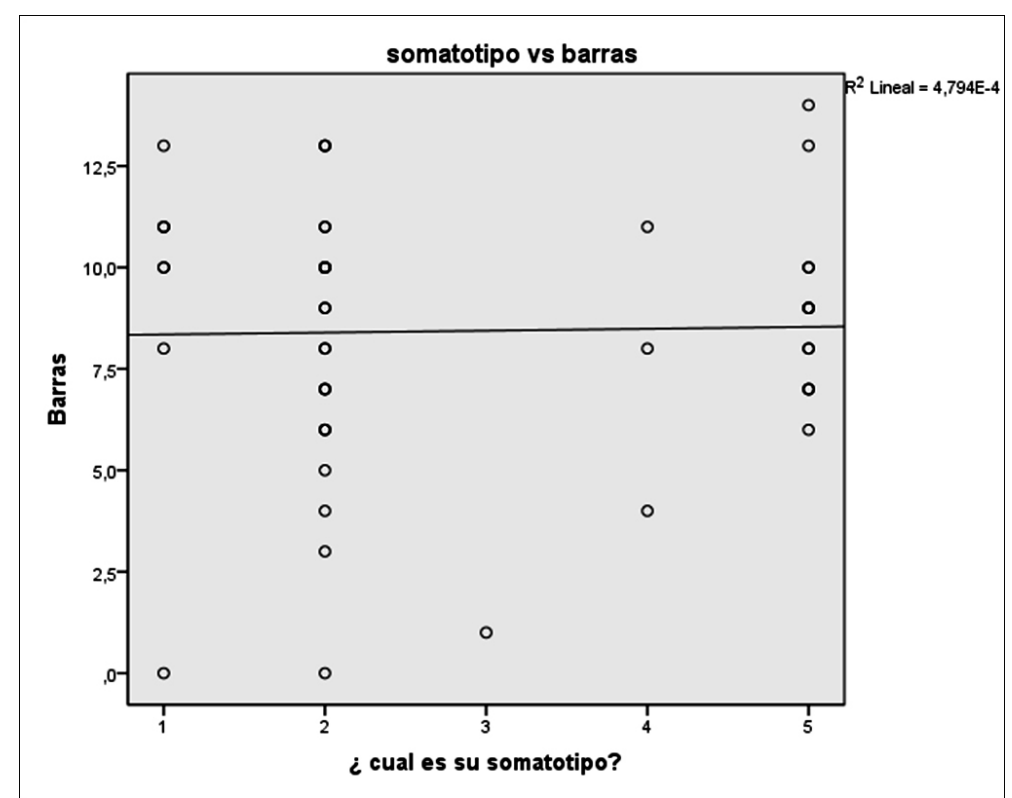

Figura 11. Somatotipo y test de barras Fuente: elaboración propia. 


\section{Discusión}

En este estudio se valoraron 51 estudiantes de la Escuela Militar de Cadetes José María Córdova, analizando la relación entre el rendimiento físico y la composición corporal en cadetes y alféreces.

Fajardo (1994), menciona en su estudio desarrollado en soldados colombianos que el promedio de edad es de 20,5 años, el peso 61,3 kg y la estatura se ubicó en 1,66 cm. Dichos resultados fueron ligeramente inferiores con los encontrados en esta investigación, donde en la edad se ubicó 19,45 ańos y presentan valores superiores en la estatura 172,6 cm y la masa corporal con $68,33 \mathrm{~kg}$.

El IMC corporal en los cadetes y alféreces se ubicó en 22,97, siendo este ligeramente superior a los resultados encontrados por Bustamante (2011) en cadetes argentinos con 22,34, por su parte en el perímetro de cintura encontraron que el 100\% ( $n=289)$ de los estudiantes no presentan ningún riesgo de padecer enfermedades hipo-cinéticas; comparado con los estudiantes de la Escuela militar el 98\% ( $\mathrm{n}=49)$ de los cadetes se ubican en este mismo indicador cuando se analizan variables antropométricas relacionadas para predecir el riesgo cardiovascular y la obesidad abdominal. Resaltamos que el $2 \%(n=2)$ de los estudiantes presentan sobrepeso siendo un factor de alerta para fortalecer los hábitos de vida saludables y el acondicionamiento físico practicado.

En cuanto al porcentaje de la masa grasa (\% MG), nuestra población en estudio presentó un promedio de $11 \%$, corroborándose valores inferiores a los aportados por Kretzel y Col (2011) con 16,1 \% en policías de fuerzas especiales de Brasil. De igual manera Crawford y Col (2011) identifican un $14 \%$ de grasa en soldados de asalto aéreo del departamento de defensa de los Estados Unidos. Los valores encontrados en el presente estudio se pueden considerar normales en relación al porcentaje de grasa para la edad en proporción con los estudios en personal Militar; poseer un bajo porcentaje de grasa tiene un efecto positivo si las actividades que realiza requieren agilidad en el desplazamiento del peso corporal.

El valor medio obtenido de la masa magra (MM) en el estudio fue de $33,5 \mathrm{~kg}$ de músculo, lo que representa el 48,8 \% de la masa total del cuerpo. Estos resultados se asemejan a los obtenidos por Kretzel y Col (2011) estimando valores de MM de 33,9 kg representados en 43,8\% de la masa total del cuerpo. Factor de importancia ya que consideramos que la masa muscular es esencial para el rendimiento físico y otras características propias para el desarrollo de la labor operacional del Militar.

El somatotipo encontrado en este estudio indica que el componente predominante es el mesomórfico $(5,1)$, siendo este un indicador de preponderancia en la masa muscular en esta población. Comparando el estudio realizado por Kretzer (2011) en un grupo de policías de fuerzas espaciales de Brasil, se encontró también una característica similar en la Mesomorfia $(4,6)$, destacamos que ambos estudios marcan una tendencia alta al desarrollo muscular; así podemos inferir que dada su labor diaria el militar requiere desplazarse con cargas adicionales al peso corporal durante un tiempo, como el equipo de campaña y el armamento, los cuales son superiores a los $35 \mathrm{~kg}$. Esto indicaría la necesidad de orientar la preparación física al desarrollo muscular disminuyendo la presencia de posibles lesiones en el aparato osteo-muscular.

De igual manera, en otro estudio realizado por Carrasco y Col (2005), en jóvenes piragüistas encontró que existe predominancia del componente Mesomorfico $(4,9)$ en este grupo de 
deportistas. Los anteriores datos son muy cercanos a nuestros resultados dado que este deporte se caracteriza por el desarrollo de la fuerza, la resistencia, la influencia del tipo de entrenamiento y condiciones de la competencia.

La resistencia muscular en el tren superior en la prueba de barras evaluada por Marín (2012) indica que el promedio logrado en jóvenes rumanos es de 5,1; comparando los resultados en esta prueba los estudiantes de la Escuela Militar logran mayores resultados con 8,4, indicando un desarrollo muscular más equilibrado dado su formación militar.

Por su parte la resistencia muscular en abdominales 76,7 y flexión de codos 54,8 evidencian resultados significativos logrando ubicarse el 76,5 ( $n=39)$ y $66,7(n=34)$ respectivamente, al comparar estos resultados con otros estudios encontramos que no existe similitud en el tiempo de duración de la prueba y la batería de ejercicios aplicada en estudios realizados por Merrodan (1999), dado que se usó como base la batería de test de la Eurofit, el cual determina 30" como el parámetro temporal para estas dos pruebas, así mismo (Marín 2012) , indica que estas dos pruebas se valoran sobre 30" de duración.

Por su parte el Vo2 (ml.kg. min $_{-1}$ ) de los estudiantes de la Escuela militar se ubicó en 42,86, siendo ligeramente inferiores estos resultados si lo comparamos con el estudio realizado por Texeira (2010). En el artículo brasilero los cadetes brasileros consiguieron 44,27(ml.kg. min $_{-1}$ ). Podemos mencionar que si bien los cadetes y alféreces tienen un nivel cercano frente a los cadetes brasileros, este punto se convierte en una oportunidad de mejora, dadas las prolongadas marchas que se realizan en las labores cotidianas.

\section{Conclusiones}

El estudio permitió determinar que existe un índice de correlación positiva leve $(>30+)$, entre el rendimiento físico y la composición corporal. En los resultados encontrados la resistencia aeróbica presenta ( $\mathrm{r}$ cuadrado=,010) la resistencia muscular en abdominales ( $\mathrm{r}$ cuadrado ,002) y la flexibilidad ( $\mathrm{r}$ cuadrado $=, 032$ ). No es posible establecer una asociación predominante entre el tipo de somatotipo y la aptitud física de los estudiantes de la Escuela Militar; sin embargo se evidencia un destacado rendimiento por parte del somatotipo endo-mesomorfo, especialmente en los test que involucran una alta participación del componente muscular.

Una preparación física acorde al desarrollo de su labor y una equilibrada composición corporal le permitirán al militar contar con altos estándares para el desarrollo de las diferentes operaciones, optimizando su rendimiento y aumentando las posibilidades de éxito operacional.

Recomendamos continuar con el seguimiento puntual al rendimiento físico establecido por la Escuela Militar y acentuar el control de la composición corporal, como factor asociado al rendimiento físico y a la disminución del riesgo de presentar enfermedad cardiovascular. 


\section{Referencias}

1. Bustamante, D. (2011). Antropometría y composición corporal. Búsqueda del mejor indicador de sobrepeso en el Cadete Naval. Archivos militares, 8, 55-69.

2. Carrasco, l., Martínez, E. y Nadal, C. (2005). Perfil antropométrico, somatotipo y composición corporal en jóvenes piraguistas. Red de revistas cientificas de América Latina, el Caribe, España y Portugal, 5(20), 270-281.

3. Crawford., K., Fleishman, K., Abt, J., Sell, T., Lovalekak, M., Nagal, T., Deluzio, J., Rowe, R., McGrail, M. \& Lephard, S. (2011). Less body omporves physical and physiological performance in Army Soldiers. Medicine Military, 176, 35-43.

4. Ejército Nacional (2007). Directiva permanente de control y evaluación de la condición física. Jefatura de educación y doctrina del Ejercito-Jedoc,

5. Esparza, F. (1993). Manual de cine antropometría. España: Edición, Grupo español de cine antropometría.

6. Fajardo, E. (1994). Valoración nutricional del soldado Colombiano y propuesta de su ración de campaña. Investigación y desarrollo social, 5, 105-120.

7. Garrido R. y González M. (2004). Índice de masa corporal y composición corporal. Un estudio antropométrico de 2500 deportistas de alto nivel. Efdeportes. Revista Digital, 10(76).

8. González, J. Molina, N., Artze, D. y Bolívar, M. (1998). Evaluación morfológica y recomendación de normas para los hombres rana profesionales cubanos. Revista Cubana de Medicina Militar, 27, 51-57.

9. Guzmán, L. (2012). Manual de Cine antropometría. Armenia: Editorial Mimesis

10. Kretzer da Luz, R., Dantas de Luca, R. y Caputo, F. (2011) Perfil antropométrico e somatotipico de Policias do bope do Estado de Santa Catarina. Educação Física em Revista, 5(3), 1-14.

11. Malina, R. (1996). Tracking of physical activity and physical fitness across the lifespan. Res Q Exerc Sport, 67(3S), 48-57.

12. Marfell-jones, M., Olds, T., Steward, A. y Carter, L. (2006). International standarsds for anthropometricas assessment. Potchesfstroom (South Africa). ISAK.

13. Marín, F. (2012). The conditional motor capacities-resistance and forcé durintthe university physical education class. Science, movement and sports, 12, 406-411.

14. Marrodan, M., Callejo, M., Moreno, E. yGonzález. M, (1999). Antropometría nutricional y aptitud fisica en adolescentes urbanos de Madrid. 51, 9-15.

15. Texeira, C. y Pereira, E. (2010). Aptitud física, edad y estado nutricional en militares. Sociedad brasilera de cardiología, 94, 420-425. 